\title{
Pretreatment with atorvastatin ameliorates cobra venom factor-induced acute lung inflammation in mice
}

\author{
Jing Guo ${ }^{1,2,3}$, Min Li ${ }^{4}$, Yi Yang ${ }^{2}$, Lin Zhang ${ }^{2}$, Li-wei Zhang ${ }^{3,5}$ and Qian-yun Sun ${ }^{1,2^{*}}$ (D)
}

\begin{abstract}
Background: The complement system plays a critical role as the pathogenic factor in the models of acute lung injury due to various causes. Cobra venom factor (CVF) is a commonly used complement research tool. The CVF can cause acute inflammation in the lung by producing complement activation components. Atorvastatin (ATR) is a 3-hydroxy-3-methylglutaryl coenzyme A inhibitor approved for control of plasma cholesterol levels. This inhibitor can reduce the acute pulmonary inflammatory response. However, the ability of ATR in treating acute lung inflammation caused by complement activation is still unknown. Therefore, we investigated the effect of ATR on lung inflammation in mice induced by activation of the complement alternative pathway in this study.
\end{abstract}

Methods: ATR (10 mg/kg/day via oral gavage) was administered for 7 days before tail vein injection of CVF ( $25 \mu \mathrm{g} /$ $\mathrm{kg}$ ). On the seventh day, all mice were sacrificed $1 \mathrm{~h}$ after injection. The lung lobe, bronchoalveolar lavage fluid (BALF), and blood samples were collected. The myeloperoxidase (MPO) activity of the lung homogenate, the leukocyte cell count, and the protein content of BALF were measured. The levels of interleukin-6 (IL-6), tumor necrosis factor-a (TNF-a), P-selectin, and Intercellular cell adhesion molecule-1 (ICAM-1) in BALF and serum were determined by enzyme-linked immunosorbent assay. The pathological change of the lung tissue was observed by hematoxylin and eosin staining. The deposition of C5b-9 in the lung tissue was detected by immunohistochemistry. The phosphorylation of NF-kB p65 in the lung tissues was examined by immunohistochemistry and western blotting.

Results: The lung inflammation levels were determined by measuring the leukocyte cell numbers and protein content of BALF, the lung MPO activity, and expression and staining of the inflammatory mediators (IL-6 and TNFa), and adhesion molecules (P-selectin and ICAM-1) for lung lesion. A significant reduction in the lung inflammation levels was observed after 7 days in ATR pre-treated mice with a CVF-induced lung disease. Deposition of C5b-9 was significantly alleviated by ATR pretreatment. Early intervention with ATR significantly reduced the development of acute lung inflammation on the basis of phosphorylation of NF-kB p65 in the lung.

(Continued on next page)

\footnotetext{
* Correspondence: sunqy@hotmail.com

'State Key Laboratory of Functions and Applications of Medicinal Plants,

Guizhou Medical University, Guiyang 550014, China

${ }^{2}$ Center for Pharmacology and Bioactivity Research, The Key Laboratory of

Chemistry for Natural Products of Guizhou Province and Chinese Academy

of Sciences, Guiyang 550014, China

Full list of author information is available at the end of the article
}

\section{$\triangle B M C$}

(c) The Author(s). 2020 Open Access This article is licensed under a Creative Commons Attribution 4.0 International License, which permits use, sharing, adaptation, distribution and reproduction in any medium or format, as long as you give appropriate credit to the original author(s) and the source, provide a link to the Creative Commons licence, and indicate if changes were made. The images or other third party material in this article are included in the article's Creative Commons licence, unless indicated otherwise in a credit line to the material. If material is not included in the article's Creative Commons licence and your intended use is not permitted by statutory regulation or exceeds the permitted use, you will need to obtain permission directly from the copyright holder. To view a copy of this licence, visit http://creativecommons.org/licenses/by/4.0/. The Creative Commons Public Domain Dedication waiver (http://creativecommons.org/publicdomain/zero/1.0/) applies to the data made available in this article, unless otherwise stated in a credit line to the data. 


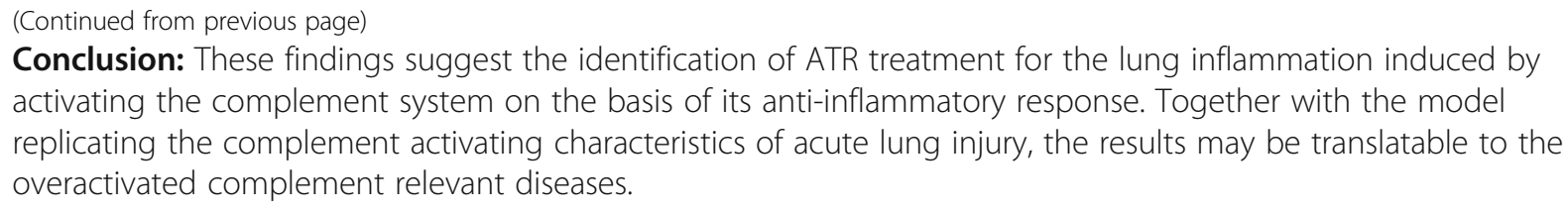

Keywords: Atorvastatin, Acute lung inflammation, Complement, Cobra venom factor, Aspirin

\section{Background}

Acute lung injury (ALI) and its severe manifestation of acute respiratory distress syndrome (ARDS) have been a major problem in humans; this condition is characterized by acute inflammation and damage of microvascular endothelial cells in the early stages [1]. Clinical [2, 3] and experimental studies $[4,5]$ have suggested the important role of complement activation products in the pathophysiology of ALI. In the early development of injury, the complement system is first activated; the abnormal or excessive activation of the complement system exhibits a startup and amplification effect in the initial stages of the inflammatory response [6, 7]. The complement system plays a critical role as the pathogenic factor in the models of acute lung injury due to various causes [8-11]. On this basis, we choose cobra venom factor (CVF), a commonly used complement research tool, to induce rapid pulmonary inflammation for exploring the pathologic changes and effective medications of lung damage. This work is conducted to truly reflect the status of pulmonary inflammation in vivo.

CVF is the protein from cobra venom that can activate complement [12]. The complement activating pattern of CVF is highly in accord with the way of complement activation in the pathological conditions of the body owing to the homology to complement component $\mathrm{C} 3$ in structure and function $[12,13]$. The CVF, as a complement experimental tool, has been extensively used to clarify its involvement in the pathogenesis of numerous diseases, including acute lung inflammation [14-17]. A previous study [17] has shown that an intravenous (i.v.) injection of CVF activated the complement alternative pathway that leads to the production of early response cytokines, such as tumor necrosis factor- $\alpha$ (TNF- $\alpha$ ) and interleukin-6 (IL-6). CVF triggers a powerful pro-inflammatory cascade that eventually leads to acute injury of the lung.

Despite the great advances in the research and therapy, ALI/ARDS mortality is still high, and therapies are scarce [18]. Statins, which are the inhibitors of 3hydroxy-3-methylglutaryl coenzyme A (HMG-CoA), are used to control the blood cholesterol levels. Recent articles [19-21] have demonstrated multipotent activities of statins, such as anti-inflammatory and anti-oxidant activities, in addition to their lipid-lowering properties; clinical evidence shows the decreasing effects of lung inflammation of statins [22, 23]. Experimental studies have shown that atorvastatin (ATR) could reverse lung injury and decrease the alveolar infiltration and proinflammatory mediator production in the initial stage of a variety of lung damage animal models [1, 22, 24]. However, the study of ATR on acute lung inflammation caused by complement activation remains unclear. In this model, the objective was to elucidate the effectiveness and mechanism of ATR treatment on an acute lung inflammation model induced by activation of the complement alternative pathway.

\section{Methods \\ Reagents}

CVF was isolated from the venom of Naja atra by our laboratory group in a previous study [25]. ATR was purchased from Jialin Pharmaceutical Incorporated Company (Beijing, China). ASA was purchased from Yunnan Baiyao Incorporated Company (Kunming, China). Carboxymethylcellulose sodium (CMC-Na) was obtained from Shanghai Aladdin Biochemical Technology Incorporated Company (Shanghai, China). The myeloperoxidase (MPO) kit was bought from Nanjing Jiancheng Bioengineering Institute (Nanjing, China). The BCA protein assay kit was obtained from Beyotime Biotechnology Co., Ltd. (Shanghai, China). Enzyme-linked immunosorbent assay (ELISA) kits for determination of mouse IL-6, TNF- $\alpha$, P-selectin, and ICAM-1 were obtained from Boster Biological Technology Co., Ltd. (Wuhan, China). A rabbit anti-C5b-9 antibody was purchased from Abcam (Cambridge, MA, USA). Antibodies for Ser 311-phosphorylated NF-kB p65 and $\beta$-actin were obtained from Santa Cruz Biotechnology (Paso Robles, CA, USA).

\section{Animals}

Thirty two Kunming mice (body weight $16 \pm 2$ g; $3-5$ weeks old; half male and half female) were provided by Hunan SJA Laboratory (Changsha, Hunan, China). Before the experiment, the animals were fed standard rodent chow and water and monitored in a controlled temperature and under a $12 \mathrm{~h} \mathrm{light/dark}$ cycle for 5 days. The experiments were performed in accordance with the protocols approved by the Institutional Animal Care and Use Committee of Guizhou Medical University (No. 1800239). 


\section{Experimental design and mouse model of ALI}

The CVF at a dose of $25 \mu \mathrm{g} / \mathrm{kg}$ body weight in sterile phosphate buffered saline (PBS, pH 7.4) was administered via tail vein injection to specifically activate the complement alternative pathway as in previous studies [17, 26]. This step was carried out to establish the mouse model of ALI. The control group received PBS. Before inducing the model, the animals were orally pretreated with ASA (aspirin $200 \mathrm{mg} / \mathrm{kg} /$ day [27], as a positive control) or ATR $(10 \mathrm{mg} / \mathrm{kg} /$ day $[21,28])$ prepared in $0.5 \%$ CMC-Na for 7 days. The vehicle-treated control group received equal volume of $0.5 \% \mathrm{CMC}-\mathrm{Na}$. On the seventh day, mice were sacrificed by an intraperitoneal (i.p.) injection of pentobarbital sodium $(50 \mathrm{mg} / \mathrm{kg}) 1 \mathrm{~h}$ after CVF or PBS challenge. Blood samples were collected in tubes via retroorbital bleed for biochemical analyses. The blood samples were left at room temperature for $1 \mathrm{~h}$ to induce clotting and centrifuged at $3000 \mathrm{r} / \mathrm{min}$ for $10 \mathrm{~min}$ at $4{ }^{\circ} \mathrm{C}$ to obtain serum. The serum was stored at $-80^{\circ} \mathrm{C}$ until used in the study.

A median sternotomy was allowed for exposure of both of the lungs. After the hilum of the right lung was ligated, the trachea was exposed and inserted with a lavage needle. Thereafter, the left lung was lavaged for four times with $0.3 \mathrm{ml}$ ice-cold saline to obtain the bronchoalveolar lavage fluid (BALF). The BALF from each sample was centrifuged at $3000 \mathrm{r} / \mathrm{min}$ and $4{ }^{\circ} \mathrm{C}$ for $10 \mathrm{~min}$ to pellet the cells after the protein content of BALF was checked with the BCA assay kit. The supernatant was stored at $-80{ }^{\circ} \mathrm{C}$ for cytokine measurement, and the resuspended cell pellet was counted with a hemocytometer. The superior lobe of the right lung was rapidly removed and stored at $-80^{\circ} \mathrm{C}$ until use. The middle lobe of the right lung was excised for the analysis of lung wet/dry weight ratio. The lower lobe of the right lung was fixed in $4 \%$ paraformaldehyde for histopathologic and immunohistochemical examination (Fig. 1).

\section{Lung wet/dry weight ratio}

The middle lobe of the right lung was cut off and weighed as wet weight, and the lung was placed in a
$70{ }^{\circ} \mathrm{C}$ incubator for at least $48 \mathrm{~h}$ to acquire the dry weight [18]. The ratio of wet to dry lung was calculated.

Lung $\mathrm{W} / \mathrm{D}$ ratio $(\%)=($ wet weight - dry weight $) /$ wet weight $\times 100 \%$

\section{MPO activity}

The lung tissues were thawed and homogenized in normal saline. The tissue homogenate was tested for the MPO level (an indicator of neutrophil accumulation [29]) with a test kit. All procedures were followed in accordance with the manufacturer's instructions.

\section{Assay of BALF and serum}

The levels of IL- 6 , TNF- $\alpha$, P-selectin, and ICAM-1 in BALF and serum were determined with ELISA kits. Meanwhile, the content of protein in BALF was estimated with the BCA protein assay kit. Total leukocyte counts were conducted under a microscope (Nikon, Tokyo, Japan) with Neubauer chambers.

\section{Histopathological examination}

The lower lobe of the right lung was dehydrated, embedded in paraffin, and cut into $5 \mu \mathrm{m}$ sections. The sections were stained with hematoxylin-eosin (H\&E) and evaluated under an optical microscope. The pathological severity score of the lung was evaluated by an investigator, who was blinded to the grouping, as previously described [30]. The scoring contents included the following four criteria: alveolar congestion, hemorrhage, infiltration of leukocytes in the lung tissue, and thickness of the alveolar wall/hyaline membrane formation. The score range was $0-4$ points $(0$, minimal damage or very mild; $1,2,3$ and 4 represented mild, moderate, severe and maximal damage, respectively.). Points were added up and the mean of total scores for the four indicators was calculated as the lung injury score.

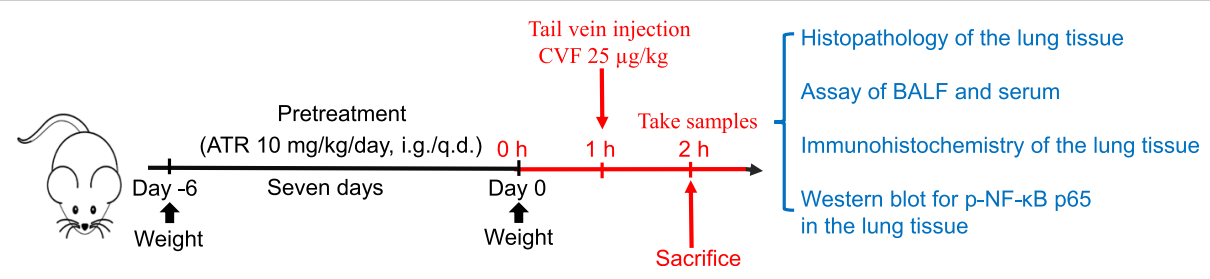

Fig. 1 Experimental timeline of the study design. Mice were weighed and grouped at day -6 . Subsequently, the mice were orally pretreated with ASA (aspirin, $200 \mathrm{mg} / \mathrm{kg} /$ day), ATR (atorvastatin, $10 \mathrm{mg} / \mathrm{kg} /$ day), or an equal volume of $0.5 \%$ CMC-Na once daily (q.d.) for 7 days until day 0 . On day 0 , the mice were weighed and given with ASA, ATR, or $0.5 \%$ CMC-Na via the intragastric route $1 \mathrm{~h}$ prior to the induction of acute lung inflammation via CVF injection (tail vein, i.v.). The control group received PBS injection. The mice ( $n=8 /$ each group) were sacrificed $1 \mathrm{~h}$ after CVF injection, and the blood, BALF, and lung tissues were collected for index measurement. CMC-Na, carboxymethylcellulose sodium; CVF, cobra venom factor; PBS, phosphate buffered saline; BALF, bronchoalveolar lavage fluid 


\section{Immunohistochemistry}

The streptavidin-biotin complex method was used to test C5b-9 and the phosphorylation of NF- $\mathrm{KB}$ p 65 in the $5 \mu \mathrm{m}$ lung sections. The lung tissue sections were stained with rabbit antibody against C5b-9 (1:100) and phospho-NF- $\kappa$ B p65 (1:500) overnight at $4{ }^{\circ} \mathrm{C}$ and incubated with horseradish peroxidase-conjugated goat antirabbit IgG antibody (Biosynthesis Biotechnology, Beijing, China) for $1 \mathrm{~h}$ at room temperature. Finally, the lung sections were stained in brown using diaminobenzidine (Beyotime Biotechnology, Shanghai, China) and then counterstained with hematoxylin. The phosphorylation level of NF- $\mathrm{kB}$ p65 was detected according to the average optical density by using Image-Pro Plus software. Scoring for C5b-9 staining was performed on the lung tissues using a $0-3$ points scoring system: 0 represented no staining, and 1, 2, and 3 denoted low, moderate, and high staining, respectively.

\section{Western blot analysis}

The tissues were homogenized, and the protein concentrations were determined with the BCA protein assay kit. The proteins $(30 \mu \mathrm{g})$ were separated on a denaturing $10 \%$ polyacrylamide gel and transferred to a polyvinylidene difluoride membrane. The following primary antibody was used: phospho-NF-kB p65 (1:1000). The membranes were then incubated with the secondary antibody at a dilution of 1:1000 (goat anti-rabbit $\operatorname{IgG}$ ) for $1 \mathrm{~h}$ at room temperature and imaged with the Vilber Fusion FX6 Spectra imaging system. The bands were quantified using VisionCapt v16.15 software (Vilber Lourmat, France). $\beta$-Actin (1:1000) was used as an internal control.

\section{Statistical analysis}

Statistical analyses of data were performed using SPSS 18.0. Data were expressed as mean \pm standard error of the mean (SEM). For lung injury scores, total cells in BALF, ICAM-1 in serum and C5b-9 deposition statistical analysis were assessed with the Mann-Whitney $U$ test. Other data analysis was conducted by using oneway analysis of variance. $P<0.05$ between two groups was considered statistically significant.

\section{Results}

\section{Histopathological findings}

We investigated the effect of ATR on the lung histopathologic change in mice with CVF challenge (Fig. 2). The histological analysis revealed that the lung injury of CVF-challenged mice was characterized by alveolar septal edema, mild expansion, congestion, and inflammatory cell infiltration. ASA and ATR pretreatment alleviated these changes. However, pretreatment with ATR had no significant difference compared with that with ASA. The results demonstrated that ATR attenuated CVF-induced lung inflammation.

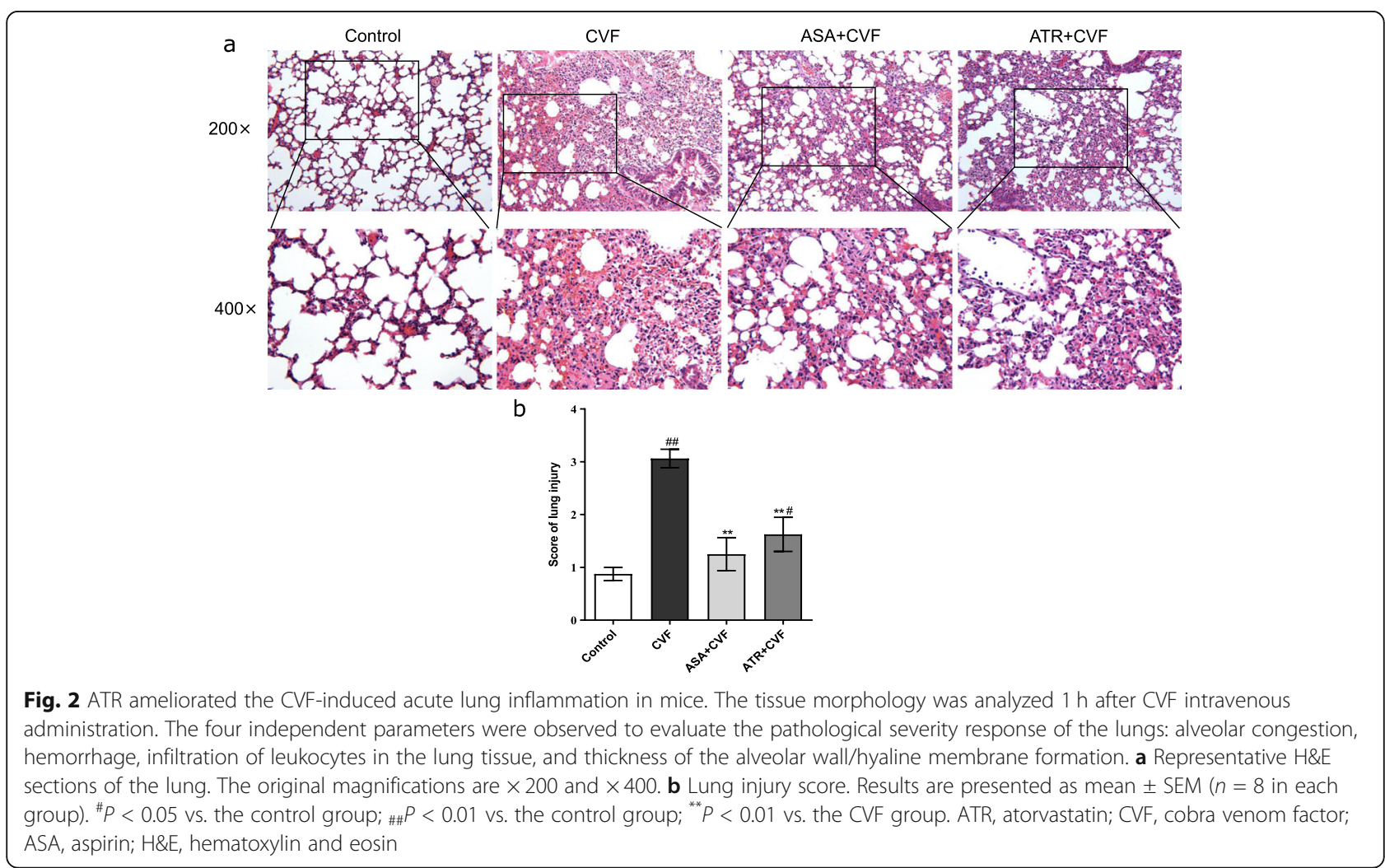




\section{Effect of ATR on neutrophil infiltration of lungs}

The accumulation of activated neutrophils in the lung is the first step in the pulmonary inflammation that leads to acute lung injury and histological damage. The total leukocyte cell counts in BALF and the MPO activity in the lung tissues were detected to investigate neutrophil activation. Figure $3 \mathrm{a}$ and $\mathrm{b}$ demonstrate that a marked increase of 63.15 and $84.22 \%$ in the total cell counts in BALF and MPO in the lung homogenate, respectively, was observed in the CVF-challenged mice compared with that in the control mice $(P<0.01)$. The ATR pretreatment significantly decreased the total cell counts and MPO level following CVF challenge to 48.12 and $81.02 \%(P<0.01)$, respectively. The increases in mice pretreatment with ASA after CVF administration were 52.63 and $84.49 \%$ compared with those in the CVF-challenged mice.

\section{Effect of ATR on the total protein level of BALF and wet to dry weight ratio of lungs}

Figure 4a illustrates that the CVF group markedly increased the protein level compared with the control group $(P<0.05)$. The pretreatment with ATR significantly reduced the protein concentration in BALF compared with the CVF group $(P<0.05)$. Figure $4 \mathrm{~b}$ shows the effect of different pretreatments on the wet to dry weight ratio of lungs. However, all groups showed no statistically significant differences.

\section{Effect of ATR on the inflammatory mediators and adhesion molecules in BALF}

Figure $5 a$ and $b$ show that the levels of IL- 6 and TNF- $\alpha$ in the CVF group were significantly increased by 65.23 and
$54.29 \%$, respectively, compared with those in the mice of the control group $(P<0.01)$. The pretreatment with ATR significantly reduced the levels of IL- 6 and TNF- $\alpha$ in BALF by 57.47 and $40.11 \%$, respectively, compared with those in the CVF group $(P<0.05$ and 0.01$)$. The levels of IL-6 and TNF- $\alpha$ in the pretreatment with ASA compared with that with ATR were reduced by 17.77 and $10.34 \%$, respectively. No statistically significant differences in the levels of Pselectin and ICAM-1 were noted in BALF from the control, CVF, ATR + CVF, and ASA + CVF groups (Fig. $5 \mathrm{c}$ and d).

\section{Effect of ATR on the inflammatory mediators and adhesion molecules in serum}

Figure $6 a-d$ exhibit the effect of ATR on the levels of IL-6, TNF- $\alpha$, P-selectin, and ICAM- 1 in serum. The levels of IL6 , TNF- $\alpha$, P-selectin, and ICAM-1 in the CVF group were significantly increased by $83.46,56.07,60.82$, and $42.99 \%$, respectively, compared with the control group $(P<0.01)$. The pretreatment with the ATR markedly reduced the levels of IL-6, TNF- $\alpha$, and ICAM-1 in serum compared with that in the CVF group with decreases of 51.18, 55.99, and $37.11 \%$, respectively $(P<0.05$ and 0.01$)$. The levels of IL- 6 and TNF- $\alpha$ in the pretreatment with ASA compared with ATR were reduced by 25.75 and $27.32 \%$, respectively. However, no significant differences were seen in the levels of IL-6, TNF- $\alpha$, P-selectin, and ICAM-1 in the serum from the ATR + CVF and ASA + CVF groups $(P>0.05)$.

\section{Effect of ATR on C5b-9 deposition of the lung tissue in mice with ALI}

We examined the deposition of C5b-9 in the lung tissue to determine whether complement activation product
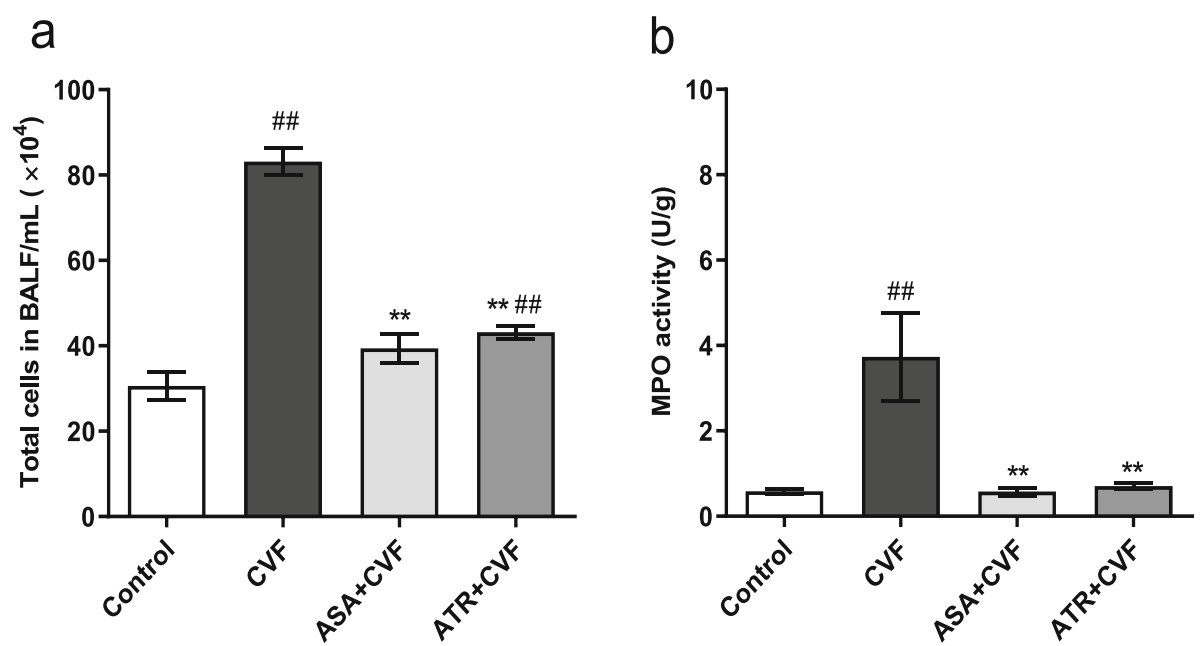

Fig. 3 ATR reduced the cell counts in the BALF and inhibited neutrophil infiltration in the lung tissues following CVF challenge. The cell counts were assessed with a hemocytometer. The superior lobe of the right lung was excised to detect the lung MPO activity $1 \mathrm{~h}$ after the CVF challenge. a Total cells in the BALF. $\mathbf{b}$ Lung MPO activity. Results are presented as mean \pm SEM ( $n=8$ in each group). ${ }^{~} P<0.05$ vs. the control group; ${ }^{\# \#} P<0.01$ vs. the control group; ${ }^{* *} P<0.01$ vs. the CVF group. ATR, atorvastatin; BALF, bronchoalveolar lavage fluid; ASA, aspirin; CVF, cobra venom factor; MPO, myeloperoxidase; SEM, standard error of the mean 
a

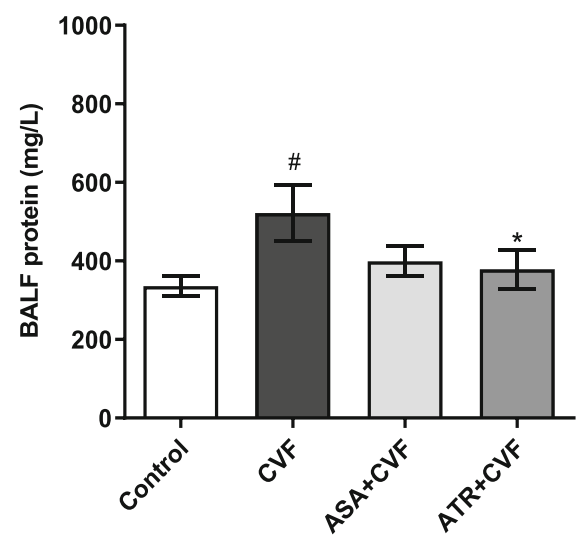

b

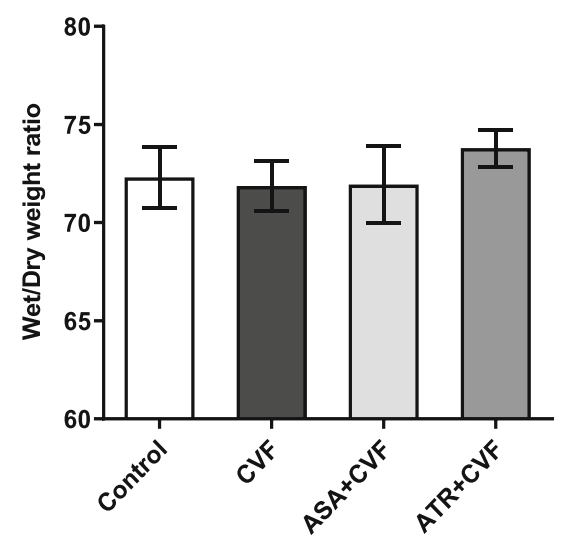

Fig. 4 ATR suppressed the total protein level of BALF and wet to dry weight ratio of lungs. a Total protein contents in the BALF. $\mathbf{b}$ Lung wet to dry weight ratio (W/D) in the middle lobe of the right lung. Results were presented as mean \pm SEM $\left(n=8\right.$ in each group). ${ }^{\# \#} P<0.01$ vs. the control group; ${ }^{*} P<0.05$ vs. the CVF group. ATR, atorvastatin; BALF, bronchoalveolar lavage fluid; CVF, cobra venom factor; SEM, standard error of the mean
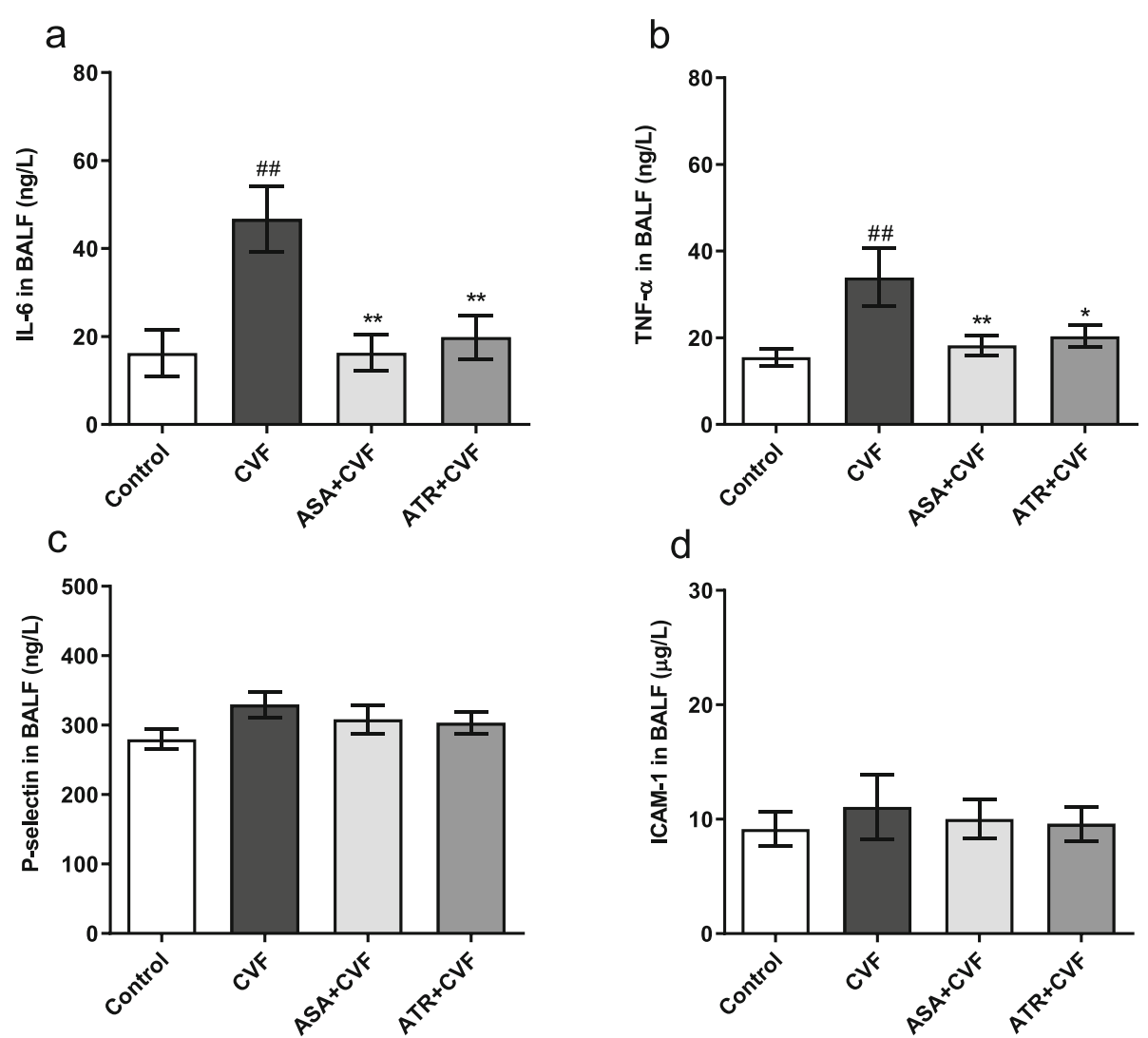

Fig. 5 ATR suppressed the inflammatory mediators and adhesion molecules in BALF. The inflammatory mediators (IL-6 and TNF-a) and adhesion molecules (ICAM-1 and P-selectin) were assessed with ELISA kits. Levels of (a) IL-6, (b) TNF-a, (c) P-selectin, and (d) ICAM-1 in BALF. Results were presented as mean \pm SEM ( $n=8$ in each group). ${ }^{\# \#} P<0.01$ vs. the control group; ${ }^{*} P<0.05$ vs. the CVF group; ${ }^{* *} P<0.01$ vs. the CVF group. ATR, atorvastatin; BALF, bronchoalveolar lavage fluid; IL-6, interleukin-6; TNF-a, tumor necrosis factor-a; ICAM-1, intercellular cell adhesion molecule-1; ELISA, enzyme-linked immunosorbent assay; CVF, cobra venom factor; SEM, standard error of the mean 

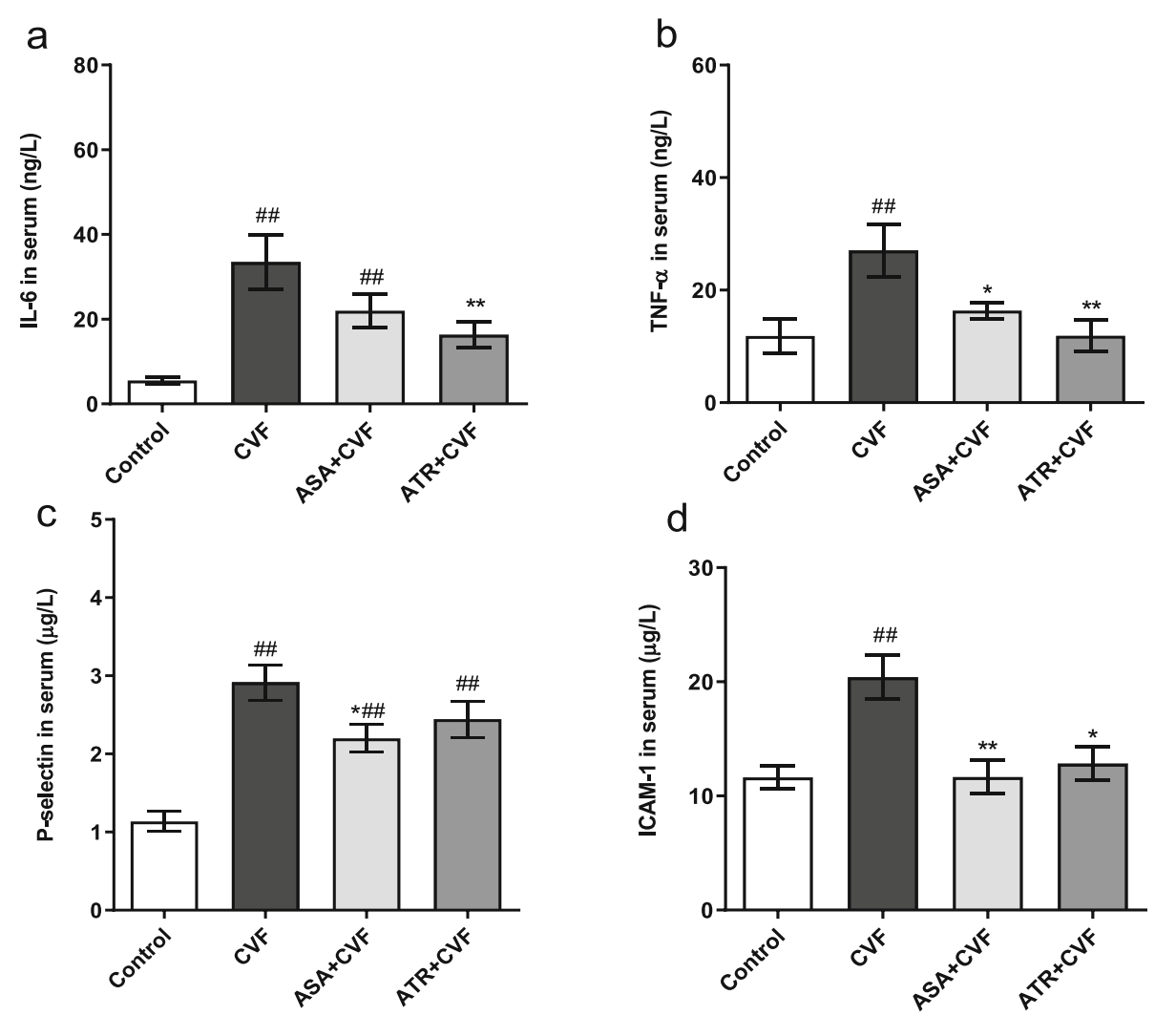

Fig. 6 ATR suppressed the inflammatory mediators and adhesion molecules in serum. The inflammatory mediators (IL-6 and TNF-a) and adhesion molecules (ICAM-1 and P-selectin) were assessed with ELISA kits. Levels of (a) IL-6, (b) TNF-a, (c) P-selectin, and (d) ICAM-1 in serum. Results were presented as mean \pm SEM ( $n=8$ in each group). ${ }^{\#} P<0.05$ Vs. the control group; ${ }^{\# \#} P<0.01$ vs. the control group; ${ }^{*} P<0.05$ Vs. the CVF group; ${ }^{* *} P<$ 0.01 vs. the CVF group. ATR, atorvastatin; BALF, bronchoalveolar lavage fluid; ASA, aspirin; IL-6, interleukin-6; TNF-a, tumor necrosis factor-a; ICAM1, intercellular cell adhesion molecule-1; ELISA, enzyme-linked immunosorbent assay; CVF, cobra venom factor; SEM, standard error of the mean

C5b-9 was inhibited by ATR after CVF injection. The results showed that the deposition of C5b-9 significantly increased after CVF injection $(P<0.01)$ (Fig. 7). The C5b-9 deposition in the lung was significantly $(P<0.05)$ reduced by $25 \%$ in mice pretreated with ATR compared with CVF mice. Meanwhile, the score for C5b-9 deposition in mice pretreated with ASA was decreased by 29.17\% compared with that in CVF mice. No significant differences on the C5b-9 deposition in the ATR + CVF group were observed compared with the ASA + CVF group $(P>0.05)$.

\section{Effect on NF-KB p65 phosphorylation in the lung tissue}

The NF- $\mathrm{kB}$ signal pathway plays an important role in the regulation of inflammation at the transcriptional level. The lung tissue sections were detected to locate phospho-NF- $\mathrm{kB}$ p65 in the lung tissue according to the positively stained cells by immunohistochemical method. This step was conducted to detect the effect of ATR on NF- $\mathrm{kB}$. Figure 8 shows that stained cells in the lung tissue of the CVF group were positively and intensively increased by $62 \%$ compared with that in the control group. The ATR + CVF group showed fewer cells positively stained in the lung tissue with a decrease of 48.42\% $(P<0.01)$ compared with the CVF group. No significant difference on the NF- $\mathrm{kB}$ p 65 phosphorylation level in the ATR + CVF group was observed compared with that in the ASA + CVF group with a decrease of 57.92\% $(P>0.05)$.

\section{Effect on phosphorylated NF-KB p65 protein expression in the lung tissue}

The western blot images of phospho-NF-kB p65 and $\beta$ actin (housekeeping) proteins in different groups are presented in Fig. 9. Consistent with the result of immunohistochemical method, the CVF significantly increased the phospho-NF- $\mathrm{KB}$ p 65 protein expression to $57.20 \%$ compared with the control mice $(P<0.01)$. The ATR + CVF group reduced the expression of phospho-NF- $\mathrm{kB}$ p65 $(P<0.01)$ with a decrease of $37.01 \%$ compared with the CVF group. No significant difference was observed in the phospho-NF-kB p65 protein expression of the ATR + CVF group compared with the ASA + CVF group with a decrease of $42.61 \% \quad(P>0.05)$. These data 

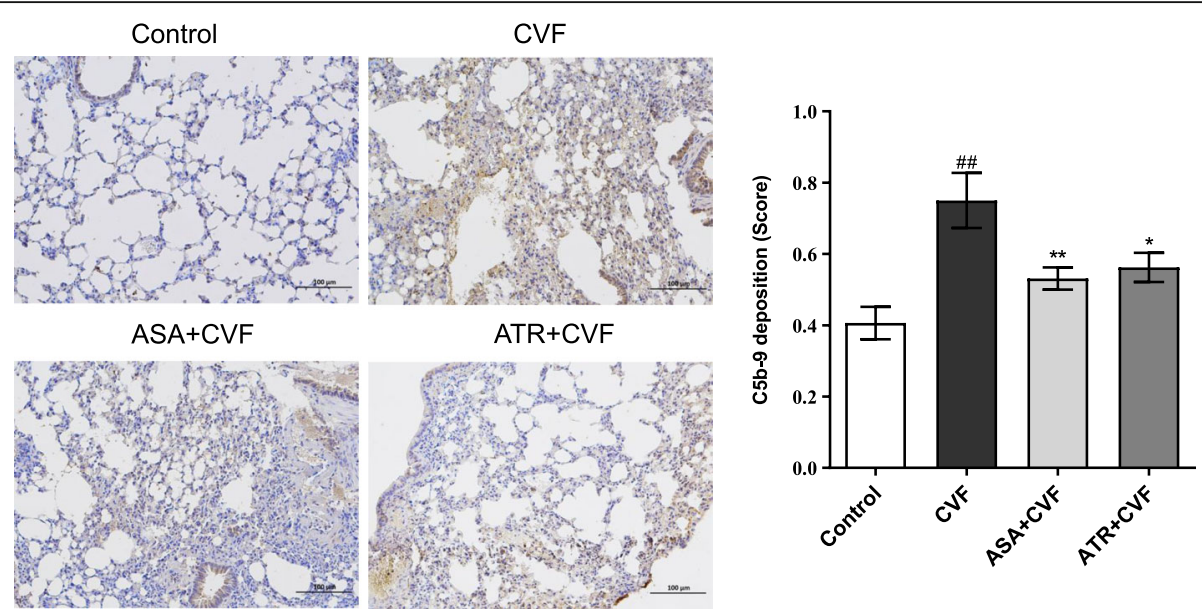

Fig. 7 ATR suppressed the C5b-9 deposition of the lung tissue in mice with ALI. The deposition of C5b-9 was determined by immunohistochemistry. The original magnification was $\times 200$. The C5b-9 deposition from the lung scores was shown as mean \pm SEM ( $n=7-8$ in each group). ${ }^{\# \#} P<0.01$ vs. the control group; ${ }^{*} P<0.05$ vs. the CVF group; ${ }^{* *} P<0.01$ vs. the CVF group. ATR, atorvastatin; CVF, cobra venom factor; ASA, aspirin; ALI, acute lung injury; SEM, standard error of the mean

suggested that ATR may inhibit the inflammatory response by downregulating the NF-kB signal pathway activation.

\section{Discussion}

Acute lung injury is a critical disease with a high incidence and mortality, and it is associated with pulmonary and systemic inflammation [23]. The complement system is one of the host defense mechanisms against infectious agents [31]. However, overactivation of the complement can lead to severe tissue damage, such as rheumatoid arthritis, sepsis, and ischemia reperfusion [6, 32]. The roles of complement in lung inflammatory injury are gradually identified [33]. Complement has been involved in the various models of acute lung injury [34-39]. Complement activation is an early step in ALI; the next complement activation products, such as $\mathrm{C} 5 \mathrm{a}$ and/or the membrane attack complex (C5b-9), can activate the neutrophils to adhere to the pulmonary capillary endothelium and directly activate endothelial cells to increase P-selectin or work with TNF- $\alpha$ to make up for the upregulation of ICAM-1 and E-selectin [33, 40]. In the process, NF-kB, a transcription factor regulating various genes involved in the inflammatory and immune responses, is activated and triggers NF- $\kappa B$ p 65 translocation to the nucleus. The augmented activation of NF- $\mathrm{kB}$ p65, a strong proinflammatory cascade, causes the transcription of inflammatory cytokines, such as TNF- $\alpha$, IL- 6 , and IL- $1 \beta$ and further results in lung injury [41]. In the complement system, three pathways can initiate the process to lead to C3
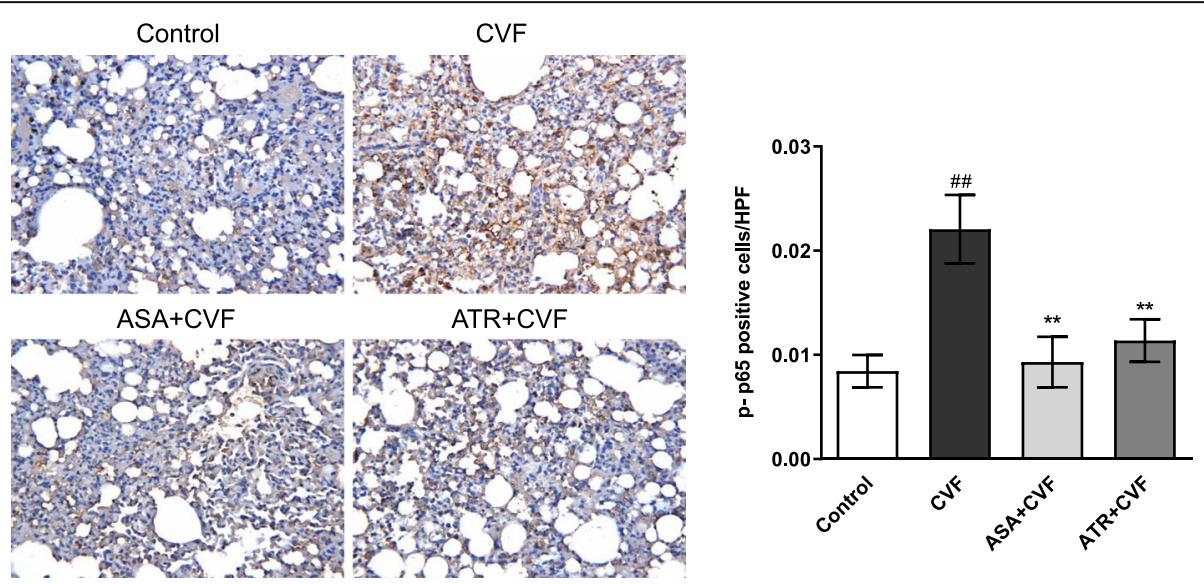

Fig. 8 ATR inhibited the expression of phosphorylated NF-KB p65 in the lung tissue. The NF-KB p65 phosphorylation was determined by immunohistochemistr and the positive cells were marked by tan or brown. Results were semiquantitatively scored by averaging the number of stained cells per high-power field. The original magnification was $\times 400$. Results were presented as mean \pm SEM $\left(n=8\right.$ in each group). ${ }^{\# \#} P<0.01$ vs. the control group; ${ }^{* *} P<0.01$ vs. the CVF group. ATR, atorvastatin; CVF, cobra venom factor; ASA, aspirin; SEM, standard error of the mean 

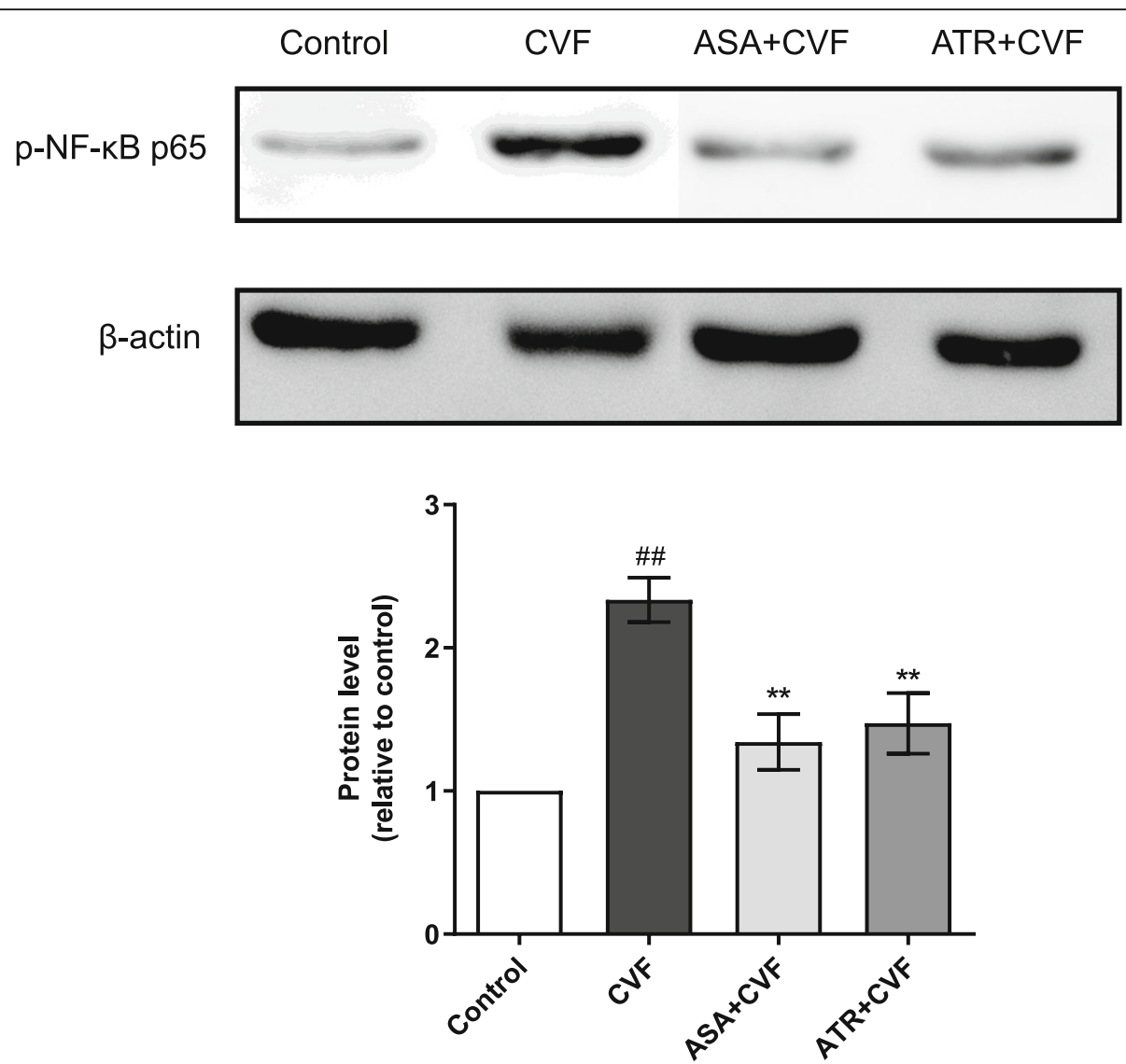

Fig. 9 ATR inhibited NF-KB activation in the lung tissue of the CVF-induced lung inflammation mice. Phosphorylated NF-KB p65 protein expression was determined by western blot. The protein expression was normalized to $\beta$-actin. Thereafter, the protein/ $\beta$-actin of the three groups $(C V F, A S A+C V F$, and ATR + CVF) was normalized to that of the control group. The protein blots were cropped on account of the presence of other groups. Results were presented as mean \pm SEM ( $n=8$ in each group). ${ }^{\# \#} P<0.01$ vs. the control group; ${ }^{* *} P<0.01$ vs. the CVF group. ATR, atorvastatin; CVF, cobra venom factor; ASA, aspirin; SEM, standard error of the mean

activation: classical, alternative, and lectin [42]. However, the three activation pathways require engagement of the alternative pathway to cause tissue injury in vivo, including acute lung inflammation [43]. Hence, the excessive activation of complement alternative plays a crucial role in the occurrence and development of acute lung inflammation.

CVF is the protein from cobra venom that can specifically activate the complement alternative pathway. This protein, a structural and functional analog of complement component $\mathrm{C} 3$, is used to study the functions of complement in immune defense and in the pathogenesis of lung injury [12, 44]. CVF can bind to factor B, and the complex is then cleaved by factor $\mathrm{D}$ to form the $\mathrm{C} 3 /$ C5 convertase. Then, the convertase generates the potent pro-inflammatory anaphylatoxins $\mathrm{C} 5 \mathrm{a}$ and $\mathrm{C} 5 \mathrm{~b}$; the latter, together with $\mathrm{C} 6-\mathrm{C} 9$, forms the complement terminal component C5b-9 [45], which can result in cell lysis [46]. Herein, we choose CVF to study the development of acute lung inflammation by activating the complement alternative pathway of mice and successfully induced the lung inflammation model. In this study, the response to acute lung inflammation was assessed by the following factors: histopathological and immunohistochemical examinations, BALF and serum levels of inflammatory mediators (IL- 6 and TNF- $\alpha$ ) and adhesion molecules (P-selectin and ICAM-1), total leukocyte cells, content of protein in BALF, W/D weight ratio, MPO activity, and NF- $\mathrm{KB}$ p 65 phosphorylation in the lung tissue. Tissue injury was histopathologically observed. The levels of the inflammatory mediators in BALF and serum were significantly increased after the induction of lung inflammation. The release of adhesion molecules in serum was markedly elevated. The phosphorylation of NF- $\mathrm{KB}$ p65 in the lung was notably upregulated in response to lung inflammation. A marked increase in the number of total cells in BALF and MPO activity in the lung homogenate and in the protein level of BALF were also observed. However, the W/D weight ratio of lungs showed no substantial change due to the unnoticeable pulmonary edema in the early stage of acute lung inflammatory lesions. In the meanwhile, complement terminal content C5b-9 of the lung tissue in the CVF- 
induced mice was significantly increased. These findings were consistent with our previous research [17]. In summary, this study found that the activation of the complement alternative pathway can induce acute lung inflammation in mice.

In the present study, we observed the effects of ATR on the lung inflammation model after activation of the complement alternative pathway in mice. Statins, which are inhibitors of 3-hydroxy-3-methylglutaryl coenzyme A reductase, were applied into clinic to lower cholesterol for minimizing cardiovascular events in the 1980s. Several publications describe the other applications of statins in anti-infective and immune modulations independent of lipid regulation [47]. The study of statins in relevant cellular and animals may also help understand the mechanism of endothelial dysfunction in acute pulmonary inflammation [23]. Jacobson et al. [48] reported that simvastatin could reduce the inflammatory parameters, such as MPO content in BALF, total neutrophil counts, and gene expression of NF-KB and IL-6 in LPS-mediated lung damage. Siempos et al. [1] demonstrated that pretreatment with ATR conferred protection against lung injury induced by high-stretch mechanical ventilation by attenuating alveolar capillary permeability. Li et al. [49] reported that simvastatin decreased the IL6 level of the lung tissue in LPS-induced septic. Ren et al. [50] reported that ATR could alleviate the lung damage and reduce mortality rate in rats with sepsis. Although simvastatin is a common statin applied in acute lung injury, the anti-inflammatory effects demonstrate differences. Melo et al. [21] evaluated the effects of ATR, pravastatin, and simvastatin on endotoxin-induced ALI and showed that ATR and pravastatin but not simvastatin revealed anti-inflammatory activity. Pinho-Ribeiro et al. [51] evaluated the effects of ATR and simvastatin on mouse lung emphysema induced by cigarette smoke. The result indicated that ATR showed a better antiinflammatory activity compared with simvastatin. The latter showed good anti-oxidant property. In combination with our search for all the studies that examined the role of statins in ARDS/ALI by China National Knowledge Internet (www.cnki.com) using the keywords ("atorvastatin" or "pravastatin") and ("acute respiratory distress syndrome" or "acute lung injury" or "acute lung inflammation" or "ARDS" or "ALI"), ATR was often used to provide a protective effect for patients who have a risk of ARDS/ALI and the animal models of pulmonary inflammation from 2010 to 2019 . However, the potential of ATR to attenuate lung inflammation due to the direct activation of complement is still unknown. Further studies of the therapeutic effects of ATR on acute lung inflammation are needed. In our study, CVF induced complement activation, inflammatory cytokine release, and adhesion molecules into the BALF and serum in addition to neutrophils and the other cells. By contrast, the ATR treatment antagonized the CVF-induced release of these mediators. The ATR pretreatment inhibited the phosphorylation of NF- $\mathrm{kB}$ p65 in the lung tissue and the deposition of C5b-9. Thus, these findings exhibited that ATR minimized the pathological lung injury induced by CVF.

We used ASA as a control drug to assess the efficacy of ATR. ASA, one of nonsteroidal anti-inflammatory drugs, has a definite anti-inflammatory effect and is used as an anti-inflammatory drug for acute and chronic inflammation. The preclinical [52-55] and clinical trials [56-61] have indicated that ASA plays a beneficial role in acute lung injury prevention and treatment. Our study indicated a similar result that pretreatment with ASA resulted in decreased pulmonary inflammatory lesions. No significant difference was observed between ASA and ATR pretreatment regarding these inflammatory outcomes.

The results showed that ATR plays a protective role against acute lung inflammation induced by activation of the complement alternative pathway. Our study extends the understanding of ATR in the development of ALI after tail vein injection of CVF. This finding indicates that ATR inhibits inflammatory cell infiltration and tissue impairment with the downregulation of adhesion molecule expression, pro-inflammatory cytokines, complement terminal product, and phosphorylation of NFкB p65.

\section{Conclusions}

This study further supports the therapeutic potential of ATR for the intervention of pulmonary inflammation. From the perspective of the complement pathogenesis, the report suggests the relationship among ATR, complement, and complement-induced diseases, such as acute lung inflammation, to a certain extent. Thus, ATR may be a novel therapeutic for complement-related diseases.

\section{Supplementary information}

Supplementary information accompanies this paper at https://doi.org/10. 1186/s12890-020-01307-3.

Additional file 1. Supplementary Information: Original western blot images.

\footnotetext{
Abbreviations

CVF: cobra venom factor; ATR: atorvastatin; BALF: bronchoalveolar lavage fluid; ALI: acute lung injury; MPO: myeloperoxidase; IL-6: interleukin-6; TNFa: tumor necrosis factor-a; ICAM-1: intercellular cell adhesion molecule-1; ARDS: acute respiratory distress syndrome; ASA: aspirin; H\&E: hematoxylin and eosin; lgG: immunoglobulin G
} 


\section{Authors' contributions}

JG and QYS conceived and designed research; YY, LZ, and LWZ participated in the design of this study; JG and ML analyzed the data; JG and QYS interpreted the results of the experiments and drafted the manuscript. All authors read and approved the final manuscript.

\section{Funding}

This work was supported by the National Natural Science Foundation of China (No. U1812403) and Guizhou Provincial Natural Science Foundation (No. QKHJC[2017]1115, QKHRC[2016]4018, QKHZC[2018]2828, QKHP TRC[2016]5625, and QKHPTRC[2019]5702). No funding provider had any influence on the design of the study or the collection, analysis, and interpretation of data or the content of this paper.

\section{Availability of data and materials}

The datasets generated and/or analyzed during the current study are available from the corresponding author on a reasonable request.

\section{Ethics approval and consent to participate}

The experimental animals were handled in accordance with a protocol approved by the Institutional Animal Care and Use Committee of Guizhou Medical University (No. 1800239).

\section{Consent for publication}

Not applicable.

\section{Competing interests}

The authors declare that they have no competing interests related to this publication.

\section{Author details}

'State Key Laboratory of Functions and Applications of Medicinal Plants, Guizhou Medical University, Guiyang 550014, China. ${ }^{2}$ Center for Pharmacology and Bioactivity Research, The Key Laboratory of Chemistry for Natural Products of Guizhou Province and Chinese Academy of Sciences, Guiyang 550014, China. ${ }^{3}$ Modern Research Center for Traditional Chinese Medicine, Shanxi University, Taiyuan 030006, China. ${ }^{4}$ General Ward, Guizhou Provincial People's Hospital, Guiyang 550002, China. ${ }^{5}$ Key Laboratory of Chemical Biology and Molecular Engineering of Education Ministry, Institute of Molecular Science, Shanxi University, Taiyuan 030006, China.

\section{Received: 31 January 2020 Accepted: 4 October 2020}

\section{Published online: 12 October 2020}

\section{References}

1. Siempos II, Maniatis NA, Kopterides P, Magkou C, Glynos C, Roussos C, Armaganidis A. Pretreatment with atorvastatin attenuates lung injury caused by high-stretch mechanical ventilation in an isolated rabbit lung model. Crit Care Med. 2010:38:1321-8.

2. Hammerschmidt DE, Weaver L, Hudson LD, Craddock PR, Jacob HS Association of complement activation and elevated plasma-C5a with adult respiratory distress syndrome. Pathophysiological relevance and possible prognostic value. Lancet. 1980;1:947-9.

3. Solomkin JS, Cotta LA, Satoh PS, Hurst JM, Nelson RD. Complement activation and clearance in acute illness and injury: evidence for C5a as a cell-directed mediator of the adult respiratory distress syndrome in man. Surgery. 1985;97:668-78.

4. Ward PA. Rous-Whipple award lecture. Role of complement in lung inflammatory injury. Am J Pathol. 1996;149:1081-6.

5. Mulligan MS, Schmid E, Beck-Schimmer B, Till GO, Friedl HP, Brauer RB, et al. Requirement and role of $\mathrm{C} 5 \mathrm{a}$ in acute lung inflammatory injury in rats. J Clin Invest. 1996:98:503-12.

6. Abe M. Complement activation and inflammation. Rinsho Byori. 2006;54 744-56.

7. Li Y, Yang Z, Chavko M, Liu B, Aderemi OA, Simovic MO, et al. Complement inhibition ameliorates blast-induced acute lung injury in rats: potential role of complement in intracellular HMGB1-mediated inflammation. PLoS One. 2018;13:e0202594.

8. Gordon T. Role of the complement system in the acute respiratory effects of inhaled endotoxin and cotton dust. Inhal Toxicol. 1994;6:253-66.
9. Greene KE, Parsons PE. Complement and endotoxin in lung injury. Acute lung injury. Springer Berlin Heidelberg;1998.p.54-69.

10. Flierl MA, Rittirsch D, Sarma JV, Huber-Lang M, Ward PA. Adrenergic regulation of complement-induced acute lung injury. Adv Exp Med Biol. 2008;632:93-103.

11. Ehrnthaller C, Ignatius A, Gebhard F, Huber-Lang M. New insights of an old defense system: structure, function, and clinical relevance of the complement system. Mol Med. 2011;17:317-29.

12. Vogel CW, Fritzinger DC. Cobra venom factor: structure, function, and humanization for therapeutic complement depletion. Toxicon. 2010;56:1198-222.

13. Till GO, Johnson KJ, Kunkel R, Ward PA. Intravascular activation of complement and acute lung injury. Dependency on neutrophils and toxic oxygen metabolites. J Clin Invest. 1982;69:1126-35.

14. Ward PA, Till GO, Hatherill JR, Annesley TM, Kunkel RG. Systemic complement activation, lung injury, and products of lipid peroxidation. J Clin Invest. 1985;76:517-27.

15. Till GO, Ward PA. Systemic complement activation and acute lung injury. Fed Proc. 1986;45:13-8.

16. Younger JG, Sasaki N, Delgado J, Ko AC, Nghiem TX, Waite MD, et al. Systemic and lung physiological changes in rats after intravascular activation of complement. J Appl Physiol. 1985;90:2289-95.

17. Guo J, Li M, Yang FM, Sun QY. Inflammatory mechanism of acute lung injury in mice induced by activation of complement alternative pathway. Chin Pharmacol Bull. 2016;32:1521-6.

18. Jiang L, Zhang L, Kang K, Fei D, Gong R, Cao Y, et al. Resveratrol ameliorates LPS-induced acute lung injury via NLRP3 inflammasome modulation. Biomed Pharmacother. 2016;84:130-8.

19. Schönbeck U, Libby P. Inflammation, immunity, and HMG-CoA reductase inhibitors: statins as antiinflammatory agents? Circulation. 2004;109:I118-26.

20. Chopra V, Flanders SA. Does statin use improve pneumonia outcomes? Chest. 2009;136:1381-8.

21. Melo AC, Valença SS, Gitirana LB, Santos JC, Ribeiro ML, Machado MN, et al. Redox markers and inflammation are differentially affected by atorvastatin pravastatin or simvastatin administered before endotoxin-induced acute lung injury. Int Immunopharmacol. 2013;17:57-64.

22. Craig T, O'Kane C, McAuley D. Potential mechanisms by which statins modulate the development of acute lung injury. Intensive Care Medicine. Springer Berlin Heidelberg: 2007.p.276-88.

23. Singla S, Jacobson JR. Statins as a novel therapeutic strategy in acute lung injury. Pulm Circ. 2012:2:397-406.

24. Souza-Costa DC, Figueiredo-Lopes L, Alves-Filho JC, Semprini MC, Gerlach $\mathrm{RF}$, Cunha FQ, Tanus-Santos JE. Protective effects of atorvastatin in rat models of acute pulmonary embolism: involvement of matrix metalloproteinase-9. Crit Care Med. 2007;35:239-45.

25. Sun QY, Chen G, Guo H, Chen S, Wang WY, Xiong YL. Prolonged cardiac xenograft survival in Guinea pig-to-rat model by a highly active cobra venom factor. Toxicon. 2003:42:257-62.

26. Proctor LM, Strachan AJ, Woodruff TM, Mahadevan IB, Williams HM, Shiels IA, Taylor SM. Complement inhibitors selectively attenuate injury following administration of cobra venom factor to rats. Int Immunopharmacol. 2006;6:1224-32.

27. Marashi SM, Hosseini SF, Hosseinzadeh M, Qadir MF, Khodaei F. Ameliorative role of aspirin in paraquat-induced lung toxicity via mitochondrial mechanisms. J Biochem Mol Toxicol. 2019:33:e22370.

28. de Paula TP, Santos PC, Arifa RDDN, et al. Treatment with atorvastatin provides additional benefits to imipenem in a model of gram-negative pneumonia induced by Klebsiella pneumoniae in mice. Antimicrob Agents Chemother. 2018;62:e00764-17.

29. Goldblum SE, Wu KM, Jay M. Lung myeloperoxidase as a measure of pulmonary leukostasis in rabbits. J Appl Physiol. 1985;59:1978-85.

30. Schingnitz U, Hartmann K, Macmanus CF, et al. Signaling through the A2B adenosine receptor dampens endotoxin-induced acute lung injury. J Immunol. 2010:184:5271-9.

31. Asghar SS. Pharmacological manipulation of the complement system in human diseases. Front Biosci. 1996:1:e15-25.

32. Sarma JV, Ward PA. New developments in C5a receptor signaling. Cell Health Cytoskelet. 2012;4:73-82.

33. Ward PA. Role of complement, chemokines, and regulatory cytokines in acute lung injury. Ann N Y Acad Sci. 1996;796:104-12.

34. Sun $S H$, Wang $H B$, Zhao GY, An YB, Du LY, Song HB, et al. A role for complement in paraquat-induced acute lung injury. Mol Immunol. 2010;47: $2212-3$. 
35. Wang RX, Xiao H, Guo RF, Li Y, Shen BF. The role of C5a in acute lung injury induced by highly pathogenic viral infections. Emerg Microbes Infect. 2015; 4:e28.

36. Sun S, Zhao G, Liu C, Wu X, Guo Y, Yu H, et al. Inhibition of complement activation alleviates acute lung injury induced by highly pathogenic avian influenza H5N1 virus infection. Am J Respir Cell Mol Biol. 2013:49:221-30.

37. Heller A, Kunz M, Samakas A, Haase M, Kirschfink M, Koch T. The complement regulators $\mathrm{C} 1$ inhibitor and soluble complement receptor 1 attenuate acute lung injury in rabbits. Shock. 2000;13:285-90.

38. Harkin DW, Marron CD, Rother RP, Romaschin A, Rubin BB, Lindsay TF. C5 complement inhibition attenuates shock and acute lung injury in an experimental model of ruptured abdominal aortic aneurysm. Br J Surg. 2005:92:1227-34

39. Mulligan MS, Smith CW, Anderson DC, Todd RF 3rd, Miyasaka M, Tamatani $\mathrm{T}$, et al. Role of leukocyte adhesion molecules in complement-induced lung injury. J Immunol. 1993;150:2401-6.

40. Bosmann M, Ward PA. Role of C3, C5 and anaphylatoxin receptors in acute lung injury and in sepsis. Adv Exp Med Biol. 2012;946:147-59.

41. Zheng H, Liang W, He W, Huang C, Chen Q, Yi H, et al. Ghrelin attenuates sepsis-induced acute lung injury by inhibiting the NF-kB, iNOS, and Akt signaling in alveolar macrophages. Am J Physiol Lung Cell Mol Physiol. 2019;317:L381-91.

42. Pandya PH, Wilkes DS. Complement system in lung disease. Am J Respir Cell Mol Biol. 2014;51:467-73.

43. Thurman JM, Holers VM. The central role of the alternative complement pathway in human disease. J Immunol. 2006;176:1305-10.

44. Tvedten HW, Till GO, Ward PA. Mediators of lung injury in mice following systemic activation of complement. Am J Pathol. 1985;119:92-100.

45. Trouw LA, Pickering MC, Blom AM. The complement system as a potential therapeutic target in rheumatic disease. Nat Rev Rheumatol. 2017;13:538-47.

46. Bayly-Jones C, Bubeck D, Dunstone MA. The mystery behind membrane insertion: a review of the complement membrane attack complex. Philos Trans R Soc Lond Ser B Biol Sci. 2017;372(1726):20160221.

47. Chalmers JD, Short PM, Mandal P, Akram AR, Hill AT. Statins in community acquired pneumonia: evidence from experimental and clinical studies. Respir Med. 2010;104:1081-91.

48. Jacobson JR, Barnard JW, Grigoryev DN, Ma SF, Tuder RM, Garcia JG. Simvastatin attenuates vascular leak and inflammation in murine inflammatory lung injury. Am J Physiol Lung Cell Mol Physiol. 2005;288: L1026-32.

49. Li WC, Zou ZJ, Zhou MG, Chen L, Zhou L, Zheng YK, He ZJ. Effects of simvastatin on the expression of inducible NOS in acute lung injury in septic rats. Int J Clin Exp Pathol. 2015:8:15106-11.

50. Ren H, Ding M, Ma H, Yao Q, Zuo G, Xu Q, et al. c. Protective effects of combined use of atorvastatin and low molecular weight heparin on the inflammatory reaction and pulmonary functions in rats with sepsis. Zhonghua Wei Zhong Bing Ji Jiu Yi Xue. 2016;28:427-32.

51. Pinho-Ribeiro V, Melo AC, Kennedy-Feitosa E, Graca-Reis A, Barroso MV, Cattani-Cavalieri I, et al. Atorvastatin and simvastatin promoted mouse lung repair after cigarette smoke-induced emphysema. Inflammation. 2017:40: 965-79.

52. Jin SW, Zhang L, Lian QQ, Liu D, Wu P, Yao SL, Ye DY. Posttreatment with aspirin-triggered lipoxin A4 analog attenuates lipopolysaccharide-induced acute lung injury in mice: the role of heme oxygenase-1. Anesth Analg. 2007:104:369-77.

53. Looney MR, Nguyen JX, Hu YM, Van Ziffle JA, Lowell CA, Matthay MA. Platelet depletion and aspirin treatment protect mice in a two-event model of transfusion-related acute lung injury. J Clin Invest. 2009;119:3450-61.

54. Tilgner J, von Trotha KT, Gombert A, Jacobs MJ, Drechsler M, Döring Y, et al. Aspirin, but not tirofiban displays protective effects in endotoxin induced lung injury. PLoS One. 2016;11:e0161218.

55. Hu X, Shen H, Wang Y, Zhang L, Zhao M. Aspirin-triggered resolvin D1 alleviates paraquat-induced acute lung injury in mice. Life Sci. 2019;218:3846.

56. Erlich JM, Talmor DS, Cartin-Ceba R, Gajic O, Kor DJ. Prehospitalization antiplatelet therapy is associated with a reduced incidence of acute lung injury: a population-based cohort study. Chest. 2011;139:289-95.

57. Kor DJ, Erlich J, Gong MN, Malinchoc M, Carter RE, Gajic O, et al. Association of prehospitalization aspirin therapy and acute lung injury: results of a multicenter international observational study of at-risk patients. Crit Care Med. 2011;39:2393-400.
58. Kor DJ, Talmor DS, Banner-Goodspeed VM, Carter RE, Hinds R, Park PK, et al. Lung injury prevention with aspirin (LIPS-A): a protocol for a multicentre randomised clinical trial in medical patients at high risk of acute lung injury. BMJ Open. 2012:2:e001606.

59. Tuinman PR, Vlaar AP, Binnenkade JM, Juffermans NP. The effect of aspirin in transfusion-related acute lung injury in critically ill patients. Anaesthesia. 2012;67:594-9.

60. Panka BA, de Grooth HJ, Spoelstra-de Man AM, Looney MR, Tuinman PR. Prevention or treatment of ARDS with aspirin: a review of preclinical models and meta-analysis of clinical studies. Shock. 2017;47:13-21.

61. Yu H, Ni YN, Liang ZA, Liang BM, Wang YM. The effect of aspirin in preventing the acute respiratory distress syndrome/acute lung injury: A meta-analysis. Am J Emerg Med. 2018;36:1486-91.

\section{Publisher's Note}

Springer Nature remains neutral with regard to jurisdictional claims in published maps and institutional affiliations.
Ready to submit your research? Choose BMC and benefit from:

- fast, convenient online submission

- thorough peer review by experienced researchers in your field

- rapid publication on acceptance

- support for research data, including large and complex data types

- gold Open Access which fosters wider collaboration and increased citations

- maximum visibility for your research: over $100 \mathrm{M}$ website views per year

At $\mathrm{BMC}$, research is always in progress.

Learn more biomedcentral.com/submissions 